\title{
Common variation contributes to the genetic architecture of social communication traits
}

\author{
Beate St. Pourcain ${ }^{1,2,3^{*}}$, Andrew J O Whitehouse ${ }^{4,5}$, Wei Q Ang ${ }^{6}$, Nicole M Warrington ${ }^{6}$, Joseph T Glessner ${ }^{7}$, \\ Kai Wang ${ }^{8}$, Nicholas J Timpson ${ }^{1,9}$, David M Evans ${ }^{1,9}$, John P Kemp ${ }^{1,9}$, Susan M Ring ${ }^{9}$, Wendy L McArdle', \\ Jean Golding ${ }^{9}$, Hakon Hakonarson ${ }^{7}$, Craig E Pennell ${ }^{6}$ and George Davey Smith ${ }^{1,9}$
}

\begin{abstract}
Background: Social communication difficulties represent an autistic trait that is highly heritable and persistent during the course of development. However, little is known about the underlying genetic architecture of this phenotype.

Methods: We performed a genome-wide association study on parent-reported social communication problems using items of the children's communication checklist (age 10 to 11 years) studying single and/or joint marker effects.

Analyses were conducted in a large UK population-based birth cohort (Avon Longitudinal Study of Parents and their Children, ALSPAC, $N=5,584$ ) and followed-up within a sample of children with comparable measures from Western Australia (RAINE, $N=1364$ ).

Results: Two of our seven independent top signals ( $P$-discovery $<1.0 \mathrm{E}-05)$ were replicated $(0.009<P$-replication $\leq 0.02)$ within RAINE and suggested evidence for association at 6p22.1 (rs9257616, meta- $P=2.5 \mathrm{E}-07$ ) and 14q22.1 (rs2352908, meta- $P=1.1 \mathrm{E}-06$ ). The signal at $6 \mathrm{p} 22.1$ was identified within the olfactory receptor gene cluster within the broader major histocompatibility complex (MHC) region. The strongest candidate locus within this genomic area was TRIM27. This gene encodes an ubiquitin E3 ligase, which is an interaction partner of methyl-CpG-binding domain (MBD) proteins, such as MBD3 and MBD4, and rare protein-coding mutations within MBD3 and MBD4 have been linked to autism. The signal at 14q22.1 was found within a gene-poor region.

Single-variant findings were complemented by estimations of the narrow-sense heritability in ALSPAC suggesting that approximately a fifth of the phenotypic variance in social communication traits is accounted for by joint additive effects of genotyped single nucleotide polymorphisms throughout the genome $\left(h^{2}(\mathrm{SE})=0.18(0.066), P=0.0027\right)$.
\end{abstract}

Conclusion: Overall, our study provides both joint and single-SNP-based evidence for the contribution of common polymorphisms to variation in social communication phenotypes.

Keywords: ALSPAC, RAINE, Autistic trait, GWAS, Social communication, Association

\section{Background}

Autism spectrum disorders (ASDs) demarcate the extreme end of a continuum of behavioural difficulties [1], characterised by impairments of social interaction and communication as well as highly restricted interests and/ or stereotyped repetitive behaviours [2]. The subthreshold end of this continuum is embodied by ASD-related but milder and non-psychopathological phenotypes, which are, as ASD, highly heritable $\left(\mathrm{h}^{2}=0.36\right.$ to 0.87 [3-9])

\footnotetext{
* Correspondence: Beate.StPourcain@bristol.ac.uk

${ }^{1}$ MRC Integrative Epidemiology Unit, University of Bristol, Oakfield House, Oakfield Grove, Bristol BS8 2BN, UK

${ }^{2}$ School of Oral and Dental Sciences, University of Bristol, Bristol, UK Full list of author information is available at the end of the article
}

and highly persistent $[10,11]$ throughout the course of development.

Twin studies have reported no difference in heritability estimates of autistic symptomatology between the extremes of the distribution and normal variation $[7,8]$, suggesting that clinical ASD and autistic-like traits in the general population may be etiologically linked. It is therefore possible that some variants influencing the expression of autistic traits might indeed represent underlying ASD quantitative trait loci (QTL). This assumption is supported by studies showing that common genetic variation at $5 \mathrm{p} 14$ [12] carries not only risk for ASD but is also associated with the expression of social

\section{Biomed Central}


communication spectrum phenotypes in the general population [13]. Candidate gene association studies identified furthermore CYP11B1 and NTRK1 as possible candidate loci, which may contribute to both risk of autism and the expression of autistic traits [14]. Twin studies, however, also suggested that there is heterogeneity among the three components of the autistic triad, and that social communication spectrum phenotypes, which are heritable traits $[6,15]$, are potentially aetiologically distinct from other autistic behavioural domains $[15,16]$.

While there are multiple efforts to investigate quantitative traits within autism samples both through linkage [17-20] and association designs [21], there is currently little known about the nature of genetic variants affecting autistic traits in the general population. The largest genome-wide effort to date has been conducted by Ronald and colleagues, using a DNA pooling approach in high- versus low-scoring individuals with respect to social and non-social autistic-like traits [22]. Although one SNP was replicated within an independent sample, the signal did not reach genome-wide significance. This might be related to some (expected) power loss because of inaccurate calls during the DNA pooling stage. Given the possibility of genetic links between the extreme and the subthreshold end of the autistic spectrum, however, a powerful genome-wide analysis of autistic traits analysed dimensionally in the general population may provide an opportunity to gain insights into the common genetic architecture of the autistic dimension. This is important, as common genetic variation identified by genome-wide association studies (GWAS) in ASD samples [12,23-27] has so far been either not replicated in more than one study [28], or did not reach evidence for genome-wide significance. Analyses of joint SNP effects suggested furthermore that the effect of common variation on risk for ASD is modest [24], highlighting the importance of study power, while other studies suggested that the lack of replication might be partially due to the underlying genetic heterogeneity of ASD, which in turn might be linked to different ASD subtypes [21]. In this context, it seems surprising that the effect of a common ASD GWAS signal at 5p14 [12] could be detected within a large population-based cohort investigating a continuum of broader ASD-related traits [13]. However, cohort designs encompass considerable advantages that can assist in the discovery of common genetic variation: cohort samples are in general large and thus highly powerful study populations, they are robust towards the influence of rare mutations of large effects and trait information can be uniformly assessed with validated instruments across an entire continuum, including both the sub-threshold end and the affected extreme.

Our study aimed to identify common variation in social communication spectrum phenotypes in the general population using GWAS. Association signals were discovered within a large UK population-based birth cohort, the Avon Longitudinal Study of Parents and their Children (ALSPAC) for which the continuity of ASD-related traits has been demonstrated [29,30], and followed-up in the Western Australian Pregnancy Cohort (RAINE) Study. Here we report support for single SNP association at 6p22.1 and 14q22.1 based on replication in independent samples.

\section{Methods}

\section{Study populations}

$A L S P A C$ is a population-based longitudinal pregnancyascertained birth cohort in the Bristol area of the UK, with an estimated date of birth between 1 April 1991 and 31 December 1992 [31]. The initial cohort included 14,541 pregnancies and additional children eligible using the original enrolment definition were recruited up to the age of 18 years, increasing the total number of pregnancies to 15,247 . The cohort is representative of the general population (approximately 96\% white mothers, based on self-report). Information on the children from these pregnancies is available from questionnaires, clinical assessments, linkage to health and administrative records as well as biological samples. Ethical approval was obtained from the ALSPAC Law and Ethics Committee (IRB00003312) and the Local Research Ethics Committees, and written informed consent was provided by all parents.

RAINE is a longitudinal investigation of 2,900 pregnant women and their offspring consecutively recruited from maternity units between 1989 and 1991 [32]. The inclusion criteria were (i) English language skills sufficient to understand the study demands, (ii) an expectation to deliver at King Edward Memorial Hospital $(\mathrm{KEMH})$, and (iii) an intention to remain in Western Australia to enable future follow-up of their child. Ninety percent of eligible women agreed to participate in the study. From the original cohort, 2,868 children have been followed over two decades. Participant recruitment and all follow-ups of their families were approved by the Human Ethics Committee at KEMH and/or Princess Margaret Hospital for Children in Perth. The RAINE sample is representative of the larger Australian population (88\% Caucasian). DNA samples have been collected using standardised procedures at 14 or 16 years of age. Only those children with both biological parents of White European origin, based on self-report, were included in the current analyses.

\section{Phenotype selection}

Social communication difficulties in ALSPAC children were measured at the age of 10 years based on motherreport using the 38-item pragmatic composite score of 
the Children's Communication Checklist (CCC) [33]. Moderate to high levels of heritability $\left(0.56<\mathrm{h}^{2}<1\right)$ have been demonstrated for all CCC subscales using twin analysis [34], though these estimates were partially based on twin pairs specifically selected for being at risk of language impairments and may, therefore, not represent the general population. In RAINE, social communication abilities were assessed with a 10-item RAINE-specific broader autism questionnaire [35] at 11 years of age based on parent-report. In order to enhance the similarity of the assessed traits, a short pragmatic composite score (SPC) was constructed based on an item-by-item alignment in both cohorts wherever possible (Table 1, Additional file 1: Figure S1), and consisted of six aligned items. For this, CCC items in ALSPAC were scored as 'certainly true' (0), 'somewhat true'(1), 'not true'(2), and RAINE broader autism questionnaire items as 'major problem'(0), 'minor problem' (1) or 'no problem' (2) resulting in a continuous measure reflecting social communication abilities with a possible range of 0 to 12 . Pertinent to this study, this highly left-skewed measure was reverse-coded, thus reflecting social communication problems, in order to facilitate a quantitative analysis of the SPC using a Poisson family model and right-skewed data.

The new measure was generated for analysis purposes only with the aim of capturing most of the shared variation in ALSPAC and RAINE, and has no further diagnostic implication. Furthermore, SPC-based statistical estimates obtained in both samples were only combined using meta-analytic approaches and heterogeneity between statistical estimates was closely monitored using heterogeneity statistics (see below).

The SPC (before reverse-coding) was highly positively correlated with the original pragmatic composite scale (Spearman rank-correlation: $\rho=0.78, P<0.0001$ ) and

\begin{tabular}{|c|c|c|}
\hline$\#$ & RAINE $^{a}$ & ALSPAC $^{\mathbf{b}}$ \\
\hline 1 & $\begin{array}{l}\text { Tends to talk about subjects } \\
\text { that are off topic }\end{array}$ & $\begin{array}{l}\text { Conversation with child tends to } \\
\text { go off in unexpected directions }\end{array}$ \\
\hline 2 & $\begin{array}{l}\text { Does not have the idea of the } \\
\text { need to take turns in conversation }\end{array}$ & Child talks too much \\
\hline 3 & Changes subject indiscriminately & $\begin{array}{l}\text { Child will suddenly change topic } \\
\text { of conversation }\end{array}$ \\
\hline 4 & $\begin{array}{l}\text { Does not introduce subjects } \\
\text { and topics appropriately }\end{array}$ & $\begin{array}{l}\text { Child uses terms like he or it without } \\
\text { making it clear what talking about }\end{array}$ \\
\hline 5 & $\begin{array}{l}\text { Has difficult expressing ideas } \\
\text { coherently in a sentence }\end{array}$ & $\begin{array}{l}\text { Sometimes hard to make sense } \\
\text { of what child says as it seems } \\
\text { illogical/disconnected }\end{array}$ \\
\hline 6 & $\begin{array}{l}\text { Has difficulty understanding } \\
\text { whole sentences (that is, instructions, } \\
\text { directions, general conversation) }\end{array}$ & $\begin{array}{l}\text { Child takes in just one or two } \\
\text { words in a sentence so often } \\
\text { misinterprets what was said }\end{array}$ \\
\hline
\end{tabular}

a - Selected from a 10-item broader autism questionnaire [35] in RAINE.

b - Selected from 38 items representing the pragmatic composite summary scale of the Children's Communication Checklist [33]. had sufficient internal consistency when investigated in ALSPAC (standardised Cronbach's $\alpha=0.68$ ) and in RAINE (standardised Cronbach's $\alpha=0.83$ ).

\section{Individuals with ASD in ALSPAC and RAINE}

Within ALSPAC there is a very small proportion of children with ASD, who were either identified from community paediatric records (National Health Service) or from Education Service databases for the region [36]. Specifically, there were 86 children with ASD at the age of 11 years (prevalence: 62 per 10,000 children). A total of 34 of these children were included within the current study as they were unrelated, of White European descent, and had both CCC/SPC data and genome-wide data. Within RAINE, there are 16 children with clinician diagnosed ASD [37]. Four of these individuals had both genotype and phenotype data available and were included in the current study.

\section{Genotyping and imputation}

ALSPAC children were genotyped using the Illumina HumanHap550 quad chip genotyping platform by 23andMe subcontracting for the Wellcome Trust Sanger Institute, Cambridge, UK and the Laboratory Corporation of America, Burlington, NC, USA. RAINE children were genotyped on an Illumina 660 Quad Array at the Centre for Applied Genomics, Toronto, ON, Canada.

Standard quality control methods were performed in each sample separately and have been previously described [38,39]. In brief, SNPs with a minor allele frequency (MAF) $<1 \%$, a call rate $<95 \%$ or evidence for violations of Hardy-Weinberg equilibrium were removed. Individual samples were excluded on the basis of sex mismatches, minimal or excessive heterozygosity, disproportionate levels of individual missingness, cryptic relatedness, insufficient sample replication and non-European ancestry. In both cohorts, subtle differences in population structure were adjusted for using principal components (Eigenstrat [40]) and genotypic data were imputed using MACH software [41] and phased haplotype data from HapMap CEU (Utah residents with Northern and Western European ancestry from the Centre d'Etude du Polymorphisme Humain collection) individuals (Rel22). Detailed information on genotyping and imputation is given for each cohort in the Additional file 1: Table S1. All reported linkage disequilibrium (LD) measures within this study are based on HapMap CEU (Rel22).

\section{Genetic association analysis}

For the discovery stage of the genome-wide analysis, we investigated 5,584 ALSPAC children with phenotypic information and approximately 2.5 million imputed and genotyped SNPs. The association analysis was performed using a Quasi-Poisson regression approach ('stats' $R$ 
library), which can accommodate for both over- and under-dispersion [42] during the modelling process. Specifically, the SPC was regressed on age, sex - the two most significant ancestry-informative principal components (guided by the evaluation of the respective Eigenvalues using a scree-plot) - and allele dosage. Using a base-line model (without fitting allele dosage) there was a dispersion parameter of $\phi=1.77$ in ALSPAC, which was statistically significant $(P<0.0001)$. Regression estimates $(\beta)$ for allele-dosages represent changes in logcounts of SPC score per effect allele and are reported with their standard errors (SE). SNPs with MAF $<1 \%$ and poor imputation quality $\left(\mathrm{R}^{2}<0.3\right)$ were excluded. Subsequently, a genomic-control method was applied to account for potential confounding by population stratification. Devlin and Roeder [43] have developed a method called 'Genomic control' that compensates for population stratification by correcting GWAS test statistics, which are presumed to be inflated by a factor $\lambda$ (where $\lambda$ can be estimated from a set of unlinked markers). After genomic control (GC)-correction (METAL [44]), we selected the strongest signals from independent loci for in silico replication in RAINE. Specifically, we selected a threshold of $P<1 \mathrm{E}-05$ in order to capture all signals with at least suggestive evidence for genome-wide association. These independent signals and associated LD-regions were identified using the PLINK software ([45], clump options: $\mathrm{r}^{2}=0.3, \pm 500 \mathrm{~kb}$ ). In order to assess the overall evidence for association based on all available samples, GC-corrected lead signals from the discovery stage were finally combined with the replication signals using fixed effect inverse-variance meta-analysis ('rmeta' R library), while testing for overall heterogeneity using Cochran's Q-test [46]. Within a fixed effect inverse-variance metaanalysis, evidence for association is combined across studies by computing the pooled inverse varianceweighted beta-coefficient, standard error and z-score [47]. However, in the presence of between-study heterogeneity, the evidence for association might be inflated by fixed effect meta-analysis and it is, therefore, important to test for heterogeneity between samples [47].

In addition to Quasi-Poisson regression, all lead signals were also investigated using Negative Binomial regression ('MASS' R library) to examine the robustness of our findings. Negative Binomial regression is an alternative regression technique accounting for the overdispersion of count data.

In order to prioritise observed SNP signals with evidence for replication in high LD regions, a gene-based association test (Versatile Gene-based Association Study (VEGAS) software [48]) was performed. Gene-based association was empirically assessed based on the $P$-value of all SNPs within a gene, while accounting for LD and the number of SNPs per gene [48].

\section{Estimation of the proportion of additive phenotypic variance explained by all SNPs together}

An estimation of the proportion of additive phenotypic variation explained by all SNPs together was performed using 'Genome-wide Complex Trait Analysis' (GCTA) [49]. This method captures the trait variance, which is tagged when all SNPs are considered simultaneously [49]. In this study, GCTA was performed using the full pragmatic composite scale of the CCC (adjusted by age, sex and the first principal components), which is highly correlated with the SPC measure (see above), as well as 464,311 directly genotyped SNPs. In addition, the additive genetic variation was partitioned into individual chromosomes. A quantitative GCTA of the SPC measure itself was not feasible as the measure is highly skewed and transformation was hampered by the limited number of items. Note that small changes in the reported sample numbers compared with the SPC are due to the exclusion of individuals with a relatedness of $\geq 2.5 \%$. The reason for applying a conservative threshold for the exclusion of family relatives is to avoid the possibility that phenotypic resemblance is due to shared environmental effects or causal effects, which are not tagged by SNPs but captured by pedigree information [49].

\section{Functional annotation}

SNP variation with evidence for replication was investigated in silico for the presence of coding variation [50], as well as non-coding variation with high functionality [51] as provided by the ENCODE database.

\section{Results}

\section{Genome-wide analysis}

Characteristics of the discovery and replication samples are presented in Table 2. GWAS in the ALSPAC cohort revealed an excess of association signals beyond chance while detecting little evidence for population stratification $\left(\lambda_{\mathrm{GC}} \leq 1.029\right.$; Figure 1). The strongest signal was observed at rs4218 within the myosin 1e gene (MYO1E) at 15q22.2 $(P=2.6 \mathrm{E}-08$, Table 3, Additional file 1: Figure S2) with an increase of 0.11 logcounts of social communication problems per effect allele.

Selecting the strongest signals from recent ASD GWAS, we furthermore investigated whether the allele conferring risk to ASD also increased the expression of social communication difficulties in the general population, as captured by the SPC score within the ALSPAC sample

\section{Table 2 Study characteristics}

\begin{tabular}{|c|c|c|c|c|c|c|c|}
\hline \multirow[b]{2}{*}{ Study } & \multirow[b]{2}{*}{ Stage } & \multirow[b]{2}{*}{$\mathrm{N}^{\mathrm{a}}$} & \multirow[b]{2}{*}{$\%$ Male } & \multicolumn{2}{|l|}{ SPC score } & \multicolumn{2}{|c|}{ Age in years } \\
\hline & & & & Mean(SD) & Range & Mean(SD) & Range \\
\hline ALSPAC & Discovery & 5,584 & 50.3 & $1.99(1.88)$ & $0 ; 12$ & $9.64(0.12)$ & $9.50 ; 11.00$ \\
\hline RAINE & Replication & 1,364 & 51.8 & $0.90(1.69)$ & $0 ; 11$ & $10.58(0.20)$ & $9.42 ; 12.37$ \\
\hline
\end{tabular}

a - Samples with genotypic and phenotypic data; SPC, Short pragmatic composite. 


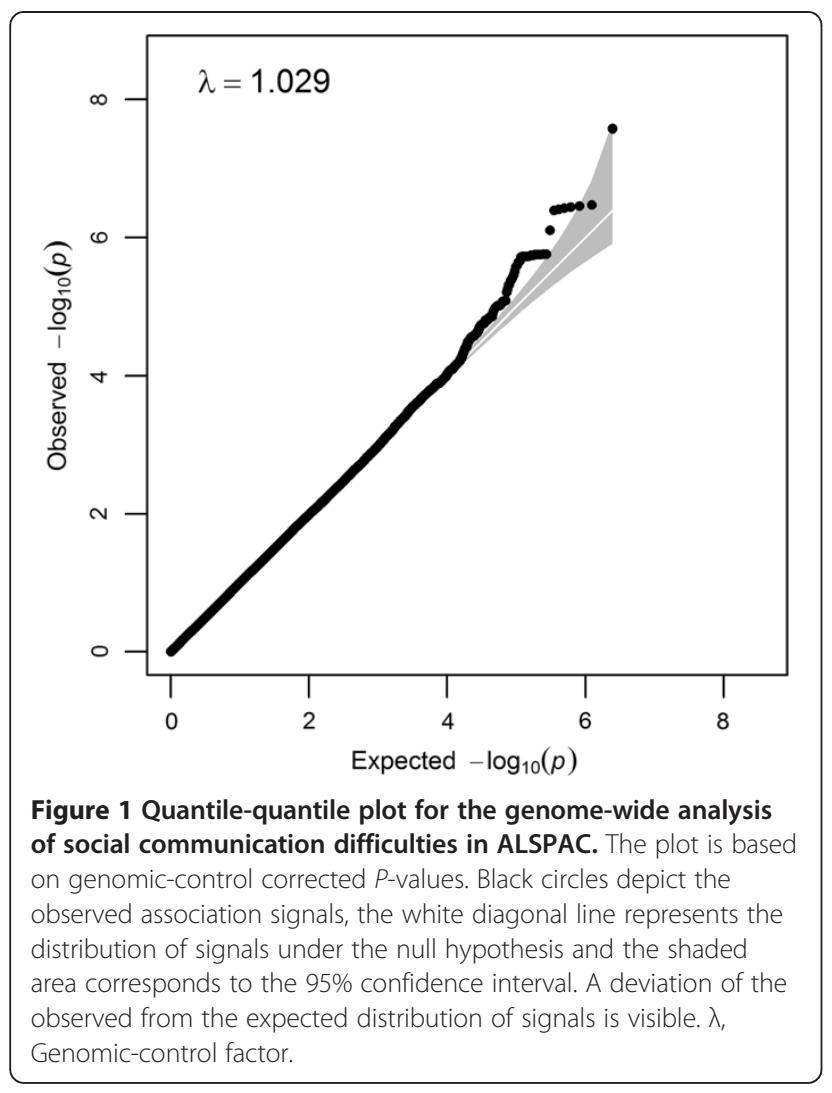

(Additional file 1: Table S2). This analysis did not identify evidence for novel ASD QTL spanning the entire spectrum, but confirmed the previously identified association between social communication traits in ALSPAC and common ASD risk variants at 5p14 [13]. Specifically, this involved the association with variation at the ASD high-risk locus rs4307059 [12] $(\beta=0.066$ (0.019), $P=$ $0.00041)$. In addition, we observed evidence for association at rs10038113 ( $\beta=-0.0391(0.018), P=0.032)$, a second ASD risk locus [26], which resides approximately $65 \mathrm{~kb}$ upstream of rs4307059 at 5p14. The association at rs4307059 was attenuated $(\beta=0.067$ (0.025), $P=0.0063)$ and the association at rs10038113 abolished $(\beta=-0.0042$ (0.023), $P=0.86$ ) when variant analyses were conditioned on each other, suggesting that these signals are not independent. Together the association findings at 5p14 thus strengthen the validity of the utilised SPC score, that is, the extent to which the SPC score captures ASD-related social communication symptoms.

In an attempt to replicate the association at rs4218 as well as six further signals from independent loci $(P<1 \mathrm{E}-$ $05)$, we investigated these variants in silico in RAINE. Two of these variants showed association with social communication problems with the same direction of effect as observed in ALSPAC (Table 3), including rs9257616 near the olfactory receptor $2 \mathrm{~J} 2$ gene (OR2J2) at 6p22.1 and rs2352908 within an intergenic interval at 14q22.1 (Figures 2 and 3 respectively). Association signals at these SNPs reached suggestive evidence for genome-wide association within the combined cohort sample, while expressing little evidence for heterogeneity: rs9257616, $\beta=$ $0.093(0.018)$, meta- $P=2.5 \mathrm{E}-07$, Het- $P=0.16$ and rs2352908, $\beta=0.12(0.025)$, meta- $P=1.1 \mathrm{E}-06$, Het $-P=$ 0.25 . Alternative statistical modelling using negative binomial regression confirmed the nature of these findings (Additional file 1: Table S3). There was however no support for an association at rs4218 in RAINE, our strongest signal from the discovery analysis $(P=0.74$, Table 3$)$.

\section{GCTA}

Further support for the contribution of common variation to the genetic architecture of social communication traits was provided through the quantification of the proportion of the phenotypic variance in pragmatic composite scores in ALSPAC, which is accounted for by all genotyped SNPs together (narrow sense heritability $\left.\mathrm{h}^{2}(\mathrm{SE})=0.18(0.066), P=0.003, \mathrm{~N}=5,244\right)$. The highly correlated CCC-based pragmatic composite score was utilised as a proxy for the SPC score (as the SPC score is

Table 3 Association results for the lead signals from the discovery analysis

\begin{tabular}{|c|c|c|c|c|c|c|c|c|c|c|c|c|}
\hline \multirow[b]{2}{*}{ SNP } & \multirow[b]{2}{*}{ Chr } & \multirow[b]{2}{*}{$E, A$} & \multirow{2}{*}{$\begin{array}{l}\text { Nearest } \\
\text { Gene }\end{array}$} & \multicolumn{3}{|c|}{ Discovery $(N=5,584)$} & \multicolumn{3}{|c|}{ Replication ( $N=1,364)$} & \multicolumn{3}{|c|}{ Combined $(\mathrm{N}=6,948)$} \\
\hline & & & & EAF & $\beta(\mathrm{SE})^{\mathrm{a}}$ & $P^{\mathrm{a}}$ & EAF & $\beta$ (SE) & $P$ & $\beta(S E)$ & $P$ & Het- $P$ \\
\hline rs761490 & $1 p 32.3$ & $C, G$ & TMEM48 & 0.24 & $0.096(0.022)$ & $9.7 \mathrm{E}-06$ & 0.23 & $-0.051(0.090)$ & 0.57 & $0.088(0.021)$ & $3.0 \mathrm{E}-05$ & 0.11 \\
\hline rs9257616 & $6 p 22.1$ & $\mathrm{G}, \mathrm{A}$ & OR2J2 & 0.56 & $0.086(0.018)$ & 3.1E-06 & 0.54 & $0.19(0.074)$ & 0.009 & $0.093(0.018)$ & $2.5 \mathrm{E}-07$ & 0.16 \\
\hline rs12115663 & $9 p 22.3$ & $C, A$ & $B N C 2$ & 0.86 & $0.13(0.027)$ & $4.1 \mathrm{E}-06$ & 0.87 & $-0.12(0.10)$ & 0.23 & $0.11(0.026)$ & $3.6 \mathrm{E}-05$ & 0.018 \\
\hline rs1834180 & $10 q 25.1$ & $A, G$ & intergenic & 0.68 & $0.10(0.020)$ & $3.3 \mathrm{E}-07$ & 0.70 & $0.033(0.079)$ & 0.68 & $0.097(0.019)$ & 4.6E-07 & 0.40 \\
\hline rs2352908 & $14 q 22.1$ & $\mathrm{G}, \mathrm{T}$ & intergenic & 0.84 & $0.11(0.026)$ & $8.2 \mathrm{E}-06$ & 0.83 & $0.24(0.10)$ & 0.022 & $0.12(0.025)$ & $1.1 \mathrm{E}-06$ & 0.25 \\
\hline rs11625667 & $14 q 24.3$ & $\mathrm{G}, \mathrm{A}$ & TMEM90A & 0.36 & $0.085(0.018)$ & 4.3E-06 & 0.35 & $-0.049(0.076)$ & 0.52 & $0.077(0.018)$ & 1.7E-05 & 0.086 \\
\hline rs4218 & $15 q 22.2$ & $G, C$ & MYO1E & 0.29 & $0.11(0.020)$ & 2.6E-08 & 0.31 & $-0.027(0.080)$ & 0.74 & $0.10(0.019)$ & $1.0 \mathrm{E}-07$ & 0.096 \\
\hline
\end{tabular}

a - Genomic-control corrected.

Results are presented for the most significant signals (Genomic-control corrected $P \leq 1 \mathrm{E}-05$ ) from independent loci during the discovery stage of the analysis. Regression estimates were obtained using Quasi-Poisson regression. All SNPs were imputed and had an imputation quality of $0.90<R^{2} \leq 0.99$ (MACH); Replicated signals are indicated in bold. E, Effect allele; A, Alternative allele; EAF, Effect allele frequency; Het- $P$, Heterogeneity $P$-value. 


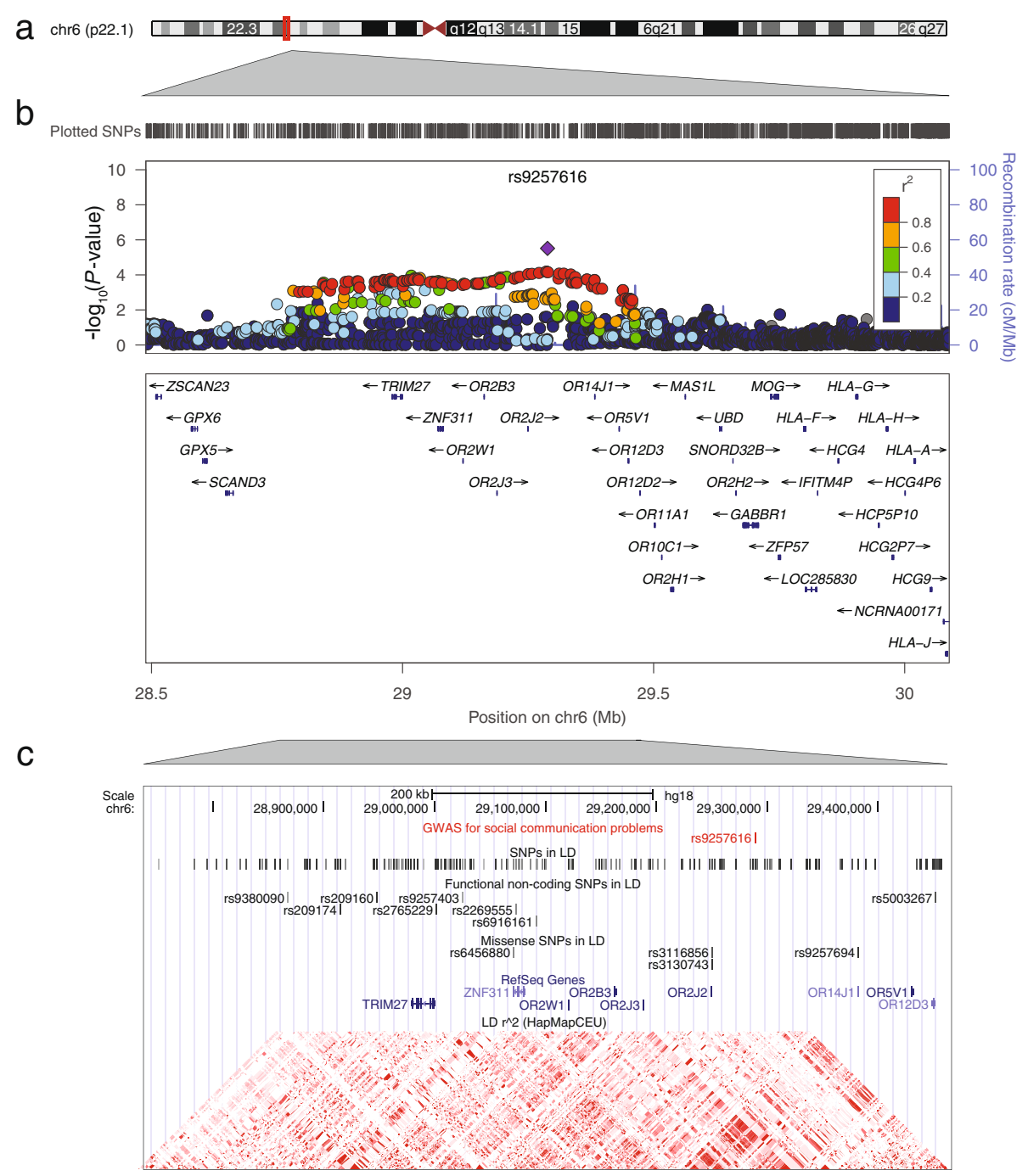

Figure 2 Association plot for the association between social communication problems and common variation at 6p22.1. a). Chromosome ideogram for chromosome 6. b). Regional association plot for rs9257616 on chromosome 6p22.1. Directly genotyped and imputed variants are depicted by filled circles according to their GWAS P-value (-log10 $P$-value) and genomic position (NCBI Build 36). The local LD structure is reflected by HapMap CEU (Rel 22) recombination rates (blue line). The LD ( $\left.r^{2}\right)$ between the lead variant and surrounding SNPs is indicated by the colour code. c). Detailed genomic region near rs9257616 on chromosome 6p22.1 with variants in LD $\left(r^{2}>0.3\right)$ including noncoding functional (Regulome score $\leq 2)$ and missense variation. The LD $\left(r^{2}\right)$ between the lead variant and surrounding SNPs is indicated by the colour code (0 (white)-1(black)). The local LD structure is reflected by HapMap CEU (Rel 22) $r^{2}$-based haplotype blocks. GWAS, genome-wide association studies; LD, linkage disequilibrium; SNPs, single nucleotide polymorphisms.

a subset of the pragmatic composite score), since the SPC measure itself could not be subjected to GCTA (see Methods).

We subsequently partitioned pragmatic composite scorerelated genetic variance into individual chromosomes, fitting all chromosomes simultaneously, and observed a trend for a linear relationship between chromosome length and explained variance supporting a polygenic inheritance model (adjusted regression $R^{2}=0.12, P=0.06$ ). However, some chromosomes, including 5,8 and 15 , may explain more phenotypic variance than predicted by the linear model (Figure 4).

\section{Annotation of functionality}

The LD structure within the vicinity of rs9257616 at 6p22.1 is complex and far reaching (LD-based gene region: approximately $707 \mathrm{~kb}$; Figure 2). Specifically, the genomic region contains a cluster of genes (TRIM27, ZNF311, OR2W1, OR2B3, OR2J3, OR2J2, LOC651503 (inferred pseudogene OR2U1P), OR214J1, OR5V1, OR12D3) among which TRIM27 provided the strongest evidence for genebased association locally (Additional file 1: Table S4). The candidacy of TRIM27 was strengthened by the presence of functional non-coding variation (Figure 2) within the vicinity of the gene (rs2765229: $\mathrm{r}^{2}=0.91$, rs9380090: $\mathrm{r}^{2}=0.41$, 


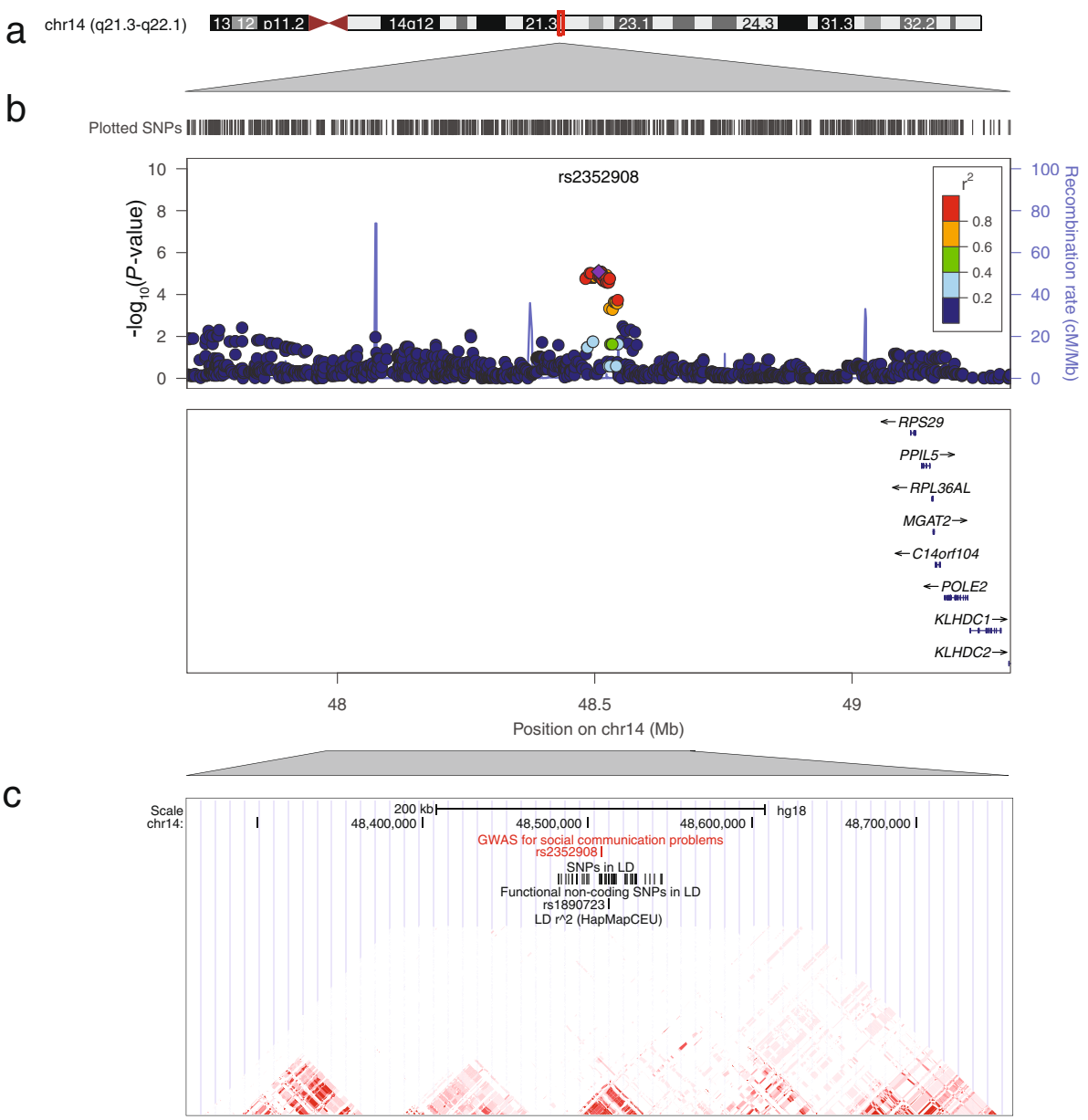

Figure 3 Association plot for the association between social communication problems and common variation at 14q22.1. a). Chromosome ideogram for chromosome 14. b). Regional association plot for rs2352908 on chromosome 14q22.1. Directly genotyped and imputed variants are depicted by filled circles according to their GWAS P-value (-log10 P-value) and genomic position (NCBI Build 36). The local LD structure is reflected by HapMap CEU (Rel 22) recombination rates (blue line). The LD $\left(r^{2}\right)$ between the lead variant and surrounding SNPs is indicated by the colour code. c). Detailed genomic region near rs2352908 on chromosome 14q22.1 with variants in LD $\left(r^{2}>0.3\right)$ including noncoding functional variation (Regulome score $\leq 2)$. The $L D\left(r^{2}\right)$ between the lead variant and surrounding SNPs is indicated by the colour code $(0$ (white)-1(black)). The local LD structure is reflected by HapMap CEU (Rel 22) $r^{2}$-based haplotype blocks. GWAS, genome-wide association studies; LD, linkage disequilibrium; SNPs, single nucleotide polymorphisms.

rs9257403: $\left.\mathrm{r}^{2}=0.43\right)$. According to the ENCODE database annotation (Additional file 1: Table S5), this variation is likely to affect the binding of various proteins, is related to histone modifications to DNA and linked to the expression of TRIM27 in monocytes. However, variation at rs9257616 was also in $\mathrm{LD}\left(\mathrm{r}^{2}>0.3\right)$ with missense mutations in OR2J2 $\left(\mathrm{rs} 3116856, \mathrm{~V}(\mathrm{GTT}) \rightarrow \mathrm{A}(\mathrm{GCT}), \mathrm{r}^{2}=0.74 ; \mathrm{rs} 3130743, \mathrm{~T}\right.$ $\left.(\mathrm{ACC}) \rightarrow \mathrm{A}(\mathrm{GCC}), \mathrm{r}^{2}=0.91\right)$, the zinc finger protein ZNF311 (rs6456880, K(AAG) $\left.\rightarrow \mathrm{Q}(\mathrm{CAG}), \mathrm{r}^{2}=0.58\right)$ and OR14J1 (rs9257694, M(ATG) $\rightarrow$ T(ACG), $\mathrm{r}^{2}=0.85$ ).

The intergenic region at $14 \mathrm{q} 22.1$ (LD-based gene region: $62 \mathrm{~kb}$; Figure 3), which harbours rs2352908, did not contain any genes within the vicinity of the signal, nor within a wider genomic region $(+/-500 \mathrm{~kb})$. The closest locus, the ribosomal protein S29 gene (RPS29) residing $606 \mathrm{~kb}$ downstream of the SNP, is separated from the variant through a recombination peak. However, there was ENCODE-based evidence for variation within a nearby functional non-coding site (rs1890723), which was in complete LD with rs2352908 $\left(\mathrm{r}^{2}=1\right)$ and linked to HNF4-based transcription regulation (Additional file 1: Table S5).

\section{Phenotypic characterisation of signals}

Analyses taking into account potential ASD-related covariates (Additional file 1: Tables S6 and S7) revealed that variation at rs2352908 was associated with an increased probability of hearing problems, in both ALSPAC (odds ratio (OR) with $\mathrm{SE}=1.48(0.24), P=$ $0.016)$ and RAINE (OR $=1.49(0.29), P=0.038)$, which 
was strongest when analyses were combined $(\mathrm{OR}=1.49$ (0.18), $P=0.0014)$. In addition, we observed weaker evidence for association between rs9257616 and internalising problems within the combined cohorts $(\mathrm{OR}=1.17$ (0.081), $P=0.022)$. Both signals were marginally attenuated when analyses were adjusted for hearing problems and internalising problems, respectively (Additional file 1 : Table S8). We found no evidence for the influence of other potential covariates, including verbal and performance intelligence quotient (IQ) scores, mother's educational level and conduct problems. Only the combined association signal between variation at rs2352908 and hearing problems would remain significant after adjustment for multiple testing.

\section{Discussion}

This genome-wide study represents a large quantitative analysis of social communication problems in the general population, analysing a total of 6,948 children of White European descent, and provided support for the implication of common variation in the genetic architecture of these traits. Two of our seven top single SNP signals at $6 \mathrm{p} 22.1$ (rs9257616, meta- $P=2.5 \mathrm{E}-07$ ) and at 14q22.1 (rs2352908, meta- $P=1.1 \mathrm{E}-06)$ were replicated within an independent sample of 11-year-old children with comparable measures from Western Australia, although they fell short of reaching conventional levels of genome-wide association. Overall, approximately a fifth (approximately 18\%) of the variation in social communication difficulties was explained by joint additive genetic effects of common SNPs (MAF $>1 \%$ ), and our findings support a polygenic mode of inheritance.

Intriguingly, the observed GCTA heritability estimates for social communication traits in the general population are highly similar to recently reported GCTA heritability estimates in relatives of ASD probands [52], strengthening the molecular support for an underlying broader autism phenotype. Based on analyses of the Simons Simplex Collection and the Autism Genome Project samples (contrasting two population control samples), substantial additive genetic influences were identified in fathers $\left(\mathrm{h}^{2}=\right.$ 0.20 to 0.52$)$, mothers $\left(\mathrm{h}^{2}=0.20\right.$ to 0.37$)$ and unaffected siblings $\left(h^{2}=0.16\right)$ [52]. The heritability estimates in our study are, however, smaller than previous twin study reports on autistic traits $\left(\mathrm{h}^{2}=0.36\right.$ to 0.87 [3-9]) as GCTA estimates reflect only the lower limit of the narrow-sense heritability and depend on the assumption that causal variation is sufficiently represented through the selected set of genotyped SNPs [49]. As such, GCTA estimates may account on average only for about half of the heritability observed within twin designs [53].

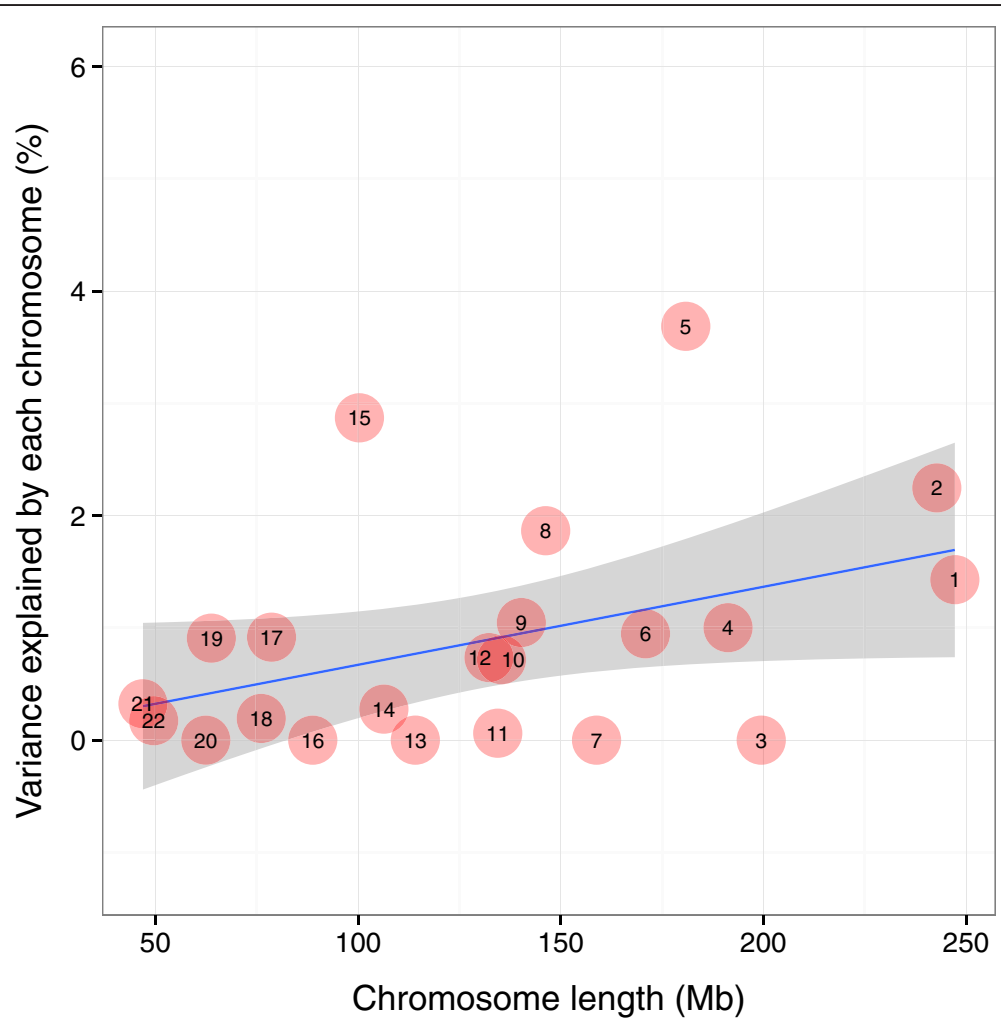

Figure 4 Estimate of the proportion of genetic variance in social communication difficulties explained by each chromosome. Numbers reflect individual chromosomes; the blue line indicates the linear regression of chromosome length on the proportion of variation explained (adjusted regression $R^{2}=0.12, P=0.06$ ); confidence intervals are indicated in grey. 
The strongest replicated single SNP signal has been identified within the olfactory receptor gene cluster at 6p22.1, which is part of the broader major histocompatibility complex $(\mathrm{MHC})$ region. On a larger scale, this genomic area has been previously related to autistic symptoms through association and linkage of the HLA-A2 class I allele with ASD [54] (approximately $768 \mathrm{~kb}$ downstream of the signal). The extensive LD across the MHC region, however, hampers the evaluation of a single locus candidacy. Both regional gene-based analysis in ALSPAC and the presence of functional non-coding variation pointed to TRIM27 (OMIM: 602165 [55]) as a candidate locus, which encodes a member of the tripartite motif (TRIM) family. TRIM27 is a DNA-binding protein associated with the nuclear matrix and interacts with methyl-CpG-binding domain (MBD) proteins [56], including MBD2, MBD3 and MBD4, and rare autism-specific protein-changing alterations have been observed both in $M B D 3$ and MBD4 [57]. Social communication related variation at $6 \mathrm{p} 22.1$ may, however, also involve one of the many OR loci or the uncharacterised ZNF311 gene, as protein altering variation at these sites has been found in LD with rs9257616. Furthermore, the replicated signals at $14 \mathrm{q} 22.1$ might be of interest as this association was supported by secondary analyses, including hearing impairments in both ALSPAC and RAINE. It might be speculated that this may reflect the nonpathological equivalent of an increased frequency of auditory symptoms, such as auditory filtering $[58,59]$ or impairment in hearing [60], which is often observed in individuals with ASD.

Partitioning of the genetic variance into chromosomes supported, furthermore, a polygenic model of inheritance, which may involve multiple loci of weak effect. This is consistent with the proposed role of common variation in ASD [24], which is likely to affect risk to disease through a (log)-additive combination of multiple loci of small effect, but also the implication of common variation within behavioural traits, such as cognitive ability [61]. It is also possible that these findings may extrapolate to other ages, with evidence from both ALSPAC [11,62] and RAINE [63] suggesting that pragmatic language skills are stable across development. However, much larger sample sizes might be required to detect loci of modest individual effects, and failure to replicate or reach conventional levels of genome-wide association may not necessarily preclude the existence of genuine (but weak) loci. In light of this, also the strongest association signals within ALSPAC, including variation at $15 \mathrm{q} 22.2$, although not replicated in the smaller RAINE sample, might be re-visited in future studies. In general, chromosome 15 harbours a large amount of common social communication related genetic variation, which is larger than expected by its size. More specifically, the signal at $15 \mathrm{q} 22.2$ was also in LD with variants at
RNF111, a gene which has been recently implicated in Asperger disorder through association [25]. However, even if this common signal is genuinely implicated in the genetic architecture of social communication traits, the underlying genetic mechanisms are likely to be different at each end of the autistic continuum, as we found no evidence that the Asperger-related single SNP variation contributes to the association signal within ALSPAC (data not shown). In addition, our findings strengthened the evidence for the presence of an ASD QTL at 5p14. Besides the signal reported by Wang and colleagues [12], which has been previously related to the expression of social communication traits in ALSPAC [13], we also observed association with a second 5p14 signal, identified by $\mathrm{Ma}$ and colleagues [26]. Conditional analysis suggested that both SNPs refer to the same underlying causal variation, thus linking both loci to the recently proposed disease mechanism involving the transcription of non-coding RNA [64].

Common genetic effects are implicated within many quantitative traits through a polygenic mode of inheritance $[61,65]$. While genome-wide genetic association screens for anthropometric phenotypes, such as height, have been, however, highly successful [65], genetic association studies involving complex behavioural traits have so far failed to robustly identify single SNP association signals $[61,66]$. Our discovery sample (Genetic power calculator; http://pngu.mgh.harvard.edu/ purcell/gpc/) had sufficient power $(>0.83)$ to detect genetic effects explaining as little as $0.7 \%$ of the phenotypic variance, assuming for simplicity a normally distributed phenotype and complete LD between marker and disease locus, in addition to a type I error of $\alpha=5 \mathrm{E}-08$. However, the true inherent power of our study might have been compromised as parent reports of social communication difficulties in children represent a far noisier and less reliable quantitative data source than comparable anthropometric phenotypes [65], making additional data cleaning and analysis steps indispensable. Within our study, we therefore selected a highly similar phenotype definition in both the discovery and the replication cohort. Problems in social communication skills as assessed by the newly defined measure are closely related to difficulties in conversational skills, such as turn taking, topic maintenance and discourse coherence. The newly defined measure had sufficient internal consistency, was highly correlated with the original CCC pragmatic composite scale [33] and consistent with a previously reported association between social communication traits and common variation at an ASD risk locus at 5p14 [13]. Furthermore, for pragmatic abilities, parent-report has been shown to be a more accurate measurement than self-report, primarily because this method allows for the assessment of communication in a variety of contexts [67]. In addition, we 
selected a Quasi-Poisson regression approach, which specifically modelled the skewed phenotypic data distribution without information loss through transformation. As such, these "power-boosting" measures may have increased the true underlying power of our study through a reduction in measurement noise. Indeed, within the specific context of GWAS of quantitative cognitive/behavioural traits our findings stand out as we identified evidence for social communication-related genetic variation through replication. However, within the general context of GWAS studies, the reported single SNPs signals reached only suggestive levels of genome-wide association and, even under the "power-boosting" circumstances, many more samples might be required to identify common genetic association signals with high confidence. Furthermore, the limited number of items that comprised the SPC $(n=6)$, may have captured only selected aspects of social communication problems. Thus, further replication efforts may require similar item alignments in order to enhance the comparability of findings across studies.

\section{Conclusion}

Our study provided evidence that common genetic variation jointly accounts for approximately a fifth of the phenotypic variation in social communication difficulties in the general population. There was furthermore support for single SNP association at $6 \mathrm{p} 22.1$ and $14 \mathrm{q} 22.1$ based on replication in independent samples, although these signals fell short of reaching conventional levels of genome-wide significance. Together our findings suggest that common genetic variation contributes to the genetic architecture of social communication traits and may indeed involve some individual loci with genetic effects large enough to be detectable in association screens.

\section{Availability of supporting data}

Supplementary information is provided as Additional material.

\section{Additional file}

Additional file 1: Table S1. Cohort-specific genotyping and imputation information. Table S2. Investigation of GWAS ASD association signals within the general population (ALSPAC) using the SPC. Table S3. Association results for the lead signals from the discovery analysis (Negative binomial regression). Table S4. Gene-based analysis of loci at 6p22.1. Table S5. Functional characterisation of non-coding variation in linkage disequilibrium with rs9257616 and rs2352908. Table S6. Association between replicated signals and potential covariates. Table S7. Association between replicated signals and intelligence. Table S8. Association for replicated lead signals with and without adjustment for potential covariates. Figure S1. Histogram of the short pragmatic composite score (SPC) in ALSPAC before reverse-coding. Figure S2. Regional association plot (Build 36) for the top 5 independent regions in the ALSPAC discovery cohort, which did not achieve replication, ordered by significance in the discovery analysis.

\section{Abbreviations}

ASD: Autism spectrum disorders; ALSPAC: Avon Longitudinal Study of Parents and their Children; CCC: Children's Communication Checklist; CNV: Copy number variation; DNA: Deoxyribonucleic acid; GC: Genomic control; GCTA: Genome-wide complex trait analysis; GWAS: Genome-wide association study; KEMH: King Edward Memorial Hospital; LD: Linkage disequilibrium; MAF: Minor allele frequency; MBD: Methyl-CpG-binding domain; MHC: Major histocompatibility complex; OR: Odds ratio;

QTL: Quantitative trait locus; SE: Standard error; SNP: Single nucleotide polymorphism; SPC score: Short Pragmatic Composite Score.

\section{Competing interests}

The authors declare that they have no competing interests.

\section{Authors' contributions}

BSP, AJOW, WQA and NMW carried out the statistical analysis. BSP, DME, JPK, SMR, WLM and NMW were involved in the preparation of the genotype information. BSP, AJOW, CEP and GDS participated in the design of the study. BSP, AJOW, WQA, JTG, KW, NJT, DMW, JPK, JG, HH, CEP and GDS helped to draft the manuscript. All authors read and approved the final manuscript.

\section{Acknowledgements}

ALSPAC: The UK Medical Research Council and the Wellcome Trust (092731), and the University of Bristol provided core support for ALSPAC, and Autism Speaks (7132) provided support for the analysis of autistic-trait related data. DME is supported by a Medical Research Council New Investigator Award (MRC G0800582). JPK is funded by a Wellcome Trust four-year PhD studentship (WT083431MA). We are extremely grateful to all the families who took part in the ALSPAC study, the midwives for their help in recruiting them, and the whole ALSPAC team, which includes interviewers, computer and laboratory technicians, clerical workers, research scientists, volunteers, managers, receptionist and nurses. We thank the Sample Logistics and Genotyping Facilities at the Wellcome Trust Sanger Institute and also 23andMe for generating the ALSPAC genome-wide data.

RAINE: The authors would like to acknowledge the National Health and Medical Research Council (NHMRC) for their long term contribution to funding the study over the last 20 years. Core Management of the RAINE study has been funded by the University of Western Australia (UWA), Curtin University, the UWA Faculty of Medicine, Dentistry and Health Sciences, the RAINE Medical Research Foundation, the Telethon Institute for Child Health Research, and the Women's and Infants Research Foundation. DNA collection and genotyping was funded by the NHMRC (572613). AJOW is funded by Career Development Fellowships from the NHMRC (1004065). The authors are extremely grateful to all of the families who took part in this study and the whole RAINE Study team, which includes the Cohort Manager, Data Manager and data collection team.

This publication is the work of the authors and they will serve as guarantors for the contents of this paper.

\section{Author details}

'MRC Integrative Epidemiology Unit, University of Bristol, Oakfield House, Oakfield Grove, Bristol BS8 2BN, UK. ${ }^{2}$ School of Oral and Dental Sciences, University of Bristol, Bristol, UK. ${ }^{3}$ School of Experimental Psychology, University of Bristol, Bristol, UK. ${ }^{4}$ Telethon Institute for Child Health Research, Centre for Child Health Research, University of Western Australia, Perth, Australia. ${ }^{5}$ School of Psychology, University of Western Australia, Perth, Australia. 'School of Women's and Infants' Health, University of Western Australia, Perth, Australia. ${ }^{7}$ Children's Hospital of Philadelphia, Philadelphia, PA, USA. ${ }^{8}$ Zilkha Neurogenetic Institute \& Department of Psychiatry, University of Southern California, Los Angeles, CA, USA. 'SChool of Social and Community Medicine, University of Bristol, Oakfield House, Oakfield Grove, Bristol BS8 2BN, UK.

Received: 16 April 2013 Accepted: 28 August 2013

Published: 18 September 2013

\section{References}

1. Wing $L$ : The continuum of autistic characteristics. In Diagnosis and Assessment in Autism. Edited by Schopler E, Mesibov G. New York, NY: Plenum; 1988:91-110. 
2. American Psychiatric Association: Diagnostic and Statistical Manual of Mental Disorders. Text Revision. 4th edition. Washington, DC: American Psychiatric Association; 2000.

3. Constantino JN, Todd RD: Autistic traits in the general population: A twin study. Arch Gen Psychiatry 2003, 60:524-530

4. Hoekstra RA, Bartels M, Verweij CJ, Boomsma Dl: Heritability of autistic traits in the general population. Arch Pediatr Adolesc Med 2007, 161:372-377.

5. Scourfield J, Martin N, Lewis G, McGuffin P: Heritability of social cognitive skills in children and adolescents. Br J Psychiatry 1999, 175:559-564.

6. Skuse D, Mandy W, Scourfield J: Measuring autistic traits: Heritability, reliability and validity of the Social and Communication Disorders Checklist. Br J Psychiatry 2005, 187:568-572.

7. Lundström S, Chang Z, Råstam M, Gillberg C, Larsson H, Anckarsäter H, Lichtenstein P: Autism spectrum disorders and autistic like traits: Similar etiology in the extreme end and the normal variation. Arch Gen Psychiatry 2012, 69:46-52.

8. Robinson EB, Koenen KC, McCormick MC, Munir K, Hallett V, Happé F, Plomin R, Ronald A: Evidence that autistic traits show the same etiology in the general population and at the quantitative extremes $(5 \%, 2.5 \%$, and 1\%). Arch Gen Psychiatry 2011, 68:1113-1121.

9. Ronald A, Happé F, Plomin R: A twin study investigating the genetic and environmental aetiologies of parent, teacher and child ratings of autisticlike traits and their overlap. Eur Child Adolesc Psychiatry 2008, 17:473-483.

10. Constantino JN, Abbacchi AM, Lavesser PD, Reed H, Givens L, Chiang L, Gray T, Gross M, Zhang Y, Todd RD: Developmental course of autistic social impairment in males. Dev Psychopathol 2009, 21:127-138.

11. St Pourcain B, Mandy WP, Heron J, Golding J, Davey Smith G, Skuse DH: Links between co-occurring social-communication and hyperactive-inattentive trait trajectories. J Am Acad Child Adolesc Psychiatry 2011, 50:892-902.

12. Wang K, Zhang H, Ma D, Bucan M, Glessner JT, Abrahams BS, Salyakina D, Imielinski M, Bradfield JP, Sleiman PM, Kim CE, Hou C, Frackelton E, Chiavacci R, Takahashi N, Sakurai T, Rappaport E, Lajonchere CM, Munson J, Estes A, Korvatska O, Piven J, Sonnenblick LI, Alvarez Retuerto Al, Herman El, Dong H, Hutman T, Sigman M, Ozonoff S, Klin A, et al: Common genetic variants on $5 \mathrm{p} 14.1$ associate with autism spectrum disorders. Nature 2009, 459:528-533.

13. St. Pourcain B, Wang K, Glessner JT, Golding J, Steer C, Ring SM, Skuse DH, Grant SFA, Hakonarson H, Davey Smith G: Association between a high-risk autism locus on $5 \mathrm{p} 14$ and social communication spectrum phenotypes in the general population. Am J Psychiatry 2010, 167:1364-1372.

14. Chakrabarti B, Dudbridge F, Kent L, Wheelwright S, Hill-Cawthorne G, Allison C, Banerjee-Basu S, Baron-Cohen S: Genes related to sex steroids, neural growth, and social-emotional behavior are associated with autistic traits, empathy, and Asperger syndrome. Autism Res 2009, 2:157-177.

15. Mandy W, Skuse D: Research review: What is the association between the social-communication element of autism and repetitive interests, behaviours and activities? J Child Psychol Psychiatry 2008, 49:795-808.

16. Ronald A, Happé F, Bolton P, Butcher LM, Price TS, Wheelwright S, BaronCohen S, Plomin R: Genetic heterogeneity between the three components of the autism spectrum: a twin study. J Am Acad Child Adolesc Psychiatry 2006, 45:691-699

17. Alarcón M, Yonan AL, Gilliam TC, Cantor RM, Geschwind DH: Quantitative genome scan and Ordered-Subsets Analysis of autism endophenotypes support language QTLs. Mol Psychiatry 2005, 10:747-757.

18. Chen GK, Kono N, Geschwind DH, Cantor RM: Quantitative trait locus analysis of nonverbal communication in autism spectrum disorder. $\mathrm{Mol}$ Psychiatry 2006, 11:214-220.

19. Duvall JA, Lu A, Cantor RM, Todd RD, Constantino JN, Geschwind DH: A quantitative trait locus analysis of social responsiveness in multiplex autism families. Am J Psychiatry 2007, 164:656-662.

20. Liu X-Q, Paterson AD, Szatmari P: Genome-wide linkage analyses of quantitative and categorical autism subphenotypes. Biol Psychiatry 2008, 64:561-570

21. Hu WW, Addington A, Hyman A: Novel autism subtype-dependent genetic variants are revealed by quantitative trait and subphenotype association analyses of published GWAS data. PLOS ONE 2011, 6:e19067.

22. Ronald A, Butcher LM, Docherty S, Davis OS, Schalkwyk LC, Craig IW, Plomin R: A genome-wide association study of social and non-social autistic-like traits in the general population using pooled DNA, $500 \mathrm{~K} \mathrm{SNP}$ microarrays and both community and diagnosed autism replication samples. Behav Genet 2010, 40:31-45.
23. Weiss LA, Arking DE, Daly MJ, Chakravarti A: A genome-wide linkage and association scan reveals novel loci for autism. Nature 2009, 461:802-808.

24. Anney R, Klei L, Pinto D, Almeida J, Bacchelli E, Baird G, Bolshakova N, Bölte S, Bolton PF, Bourgeron T, Brennan S, Brian J, Casey J, Conroy J, Correia C, Corsello C, Crawford EL, De Jonge M, Delorme R, Duketis E, Duque F, Estes A, Farrar P, Fernandez BA, Folstein SE, Fombonne E, Gilbert J, Gillberg C, Glessner JT, Green A, et al: Individual common variants exert weak effects on risk for autism spectrum disorders. Hum Mol Genet 2012, 21:4781-4792.

25. Salyakina D, Ma DQ, Jaworski JM, Konidari I, Whitehead PL, Henson R, Martinez D, Robinson JL, Sacharow S, Wright HH, Abramson RK, Gilbert JR, Cuccaro ML, Pericak-Vance MA: Variants in several genomic regions associated with asperger disorder. Autism Res 2010, 3:303-310.

26. Ma D, Salyakina D, Jaworski JM, Konidari I, Whitehead PL, Andersen AN, Hoffman JD, Slifer SH, Hedges DJ, Cukier HN, Griswold AJ, McCauley JL, Beecham GW, Wright HH, Abramson RK, Martin ER, Hussman JP, Gilbert JR, Cuccaro ML, Haines JL, Pericak-Vance MA: A genome-wide association study of autism reveals a common novel risk locus at $5 p 14.1$. Ann Human Genet 2009, 73:263-273.

27. Anney R, Klei L, Pinto D, Regan R, Conroy J, Magalhaes TR, Correia C, Abrahams BS, Sykes N, Pagnamenta AT, Almeida J, Bacchelli E, Bailey AJ, Baird G, Battaglia A, Berney T, Bolshakova N, Bölte S, Bolton PF, Bourgeron T, Brennan S, Brian J, Carson AR, Casallo G, Casey J, Chu SH, Cochrane L, Corsello C, Crawford EL, Crossett A, et al: A genome-wide scan for common alleles affecting risk for autism. Hum Mol Genet 2010, 15:4072-4082.

28. Devlin B, Melhem N, Roeder K: Do common variants play a role in risk for autism? Evidence and theoretical musings. Brain Res 2011, 1380:78-84.

29. Skuse D, Mandy W, Steer C, Miller L, Goodman R, Lawrence K, Emond A, Golding J: Social communication competence and functional adaptation in a general population of children: preliminary evidence for sex-by-verbal IQ differential risk. J Am Acad Child Adolesc Psychiatry 2008, 48:128-137.

30. Steer C, Bolton P, Roulstone S, Emond A, Golding J: Traits contributing to the autistic spectrum. PLOS ONE 2010, 5:e12633.

31. Boyd A, Golding J, Macleod J, Lawlor DA, Fraser A, Henderson J, Molloy L, Ness A, Ring S, Davey Smith G: Cohort profile: The "children of the 90s" -the index offspring of the Avon Longitudinal Study of Parents and Children. Int J Epidemiol 2013, 42:111-127.

32. Newnham JP, Evans SF, Michael CA, Stanley FJ, Landau LI: Effects of frequent ultrasound during pregnancy: a randomised controlled trial. Lancet 1993, 342:887-891.

33. Bishop DV: Development of the Children's Communication Checklist (CCC): a method for assessing qualitative aspects of communicative impairment in children. J Child Psychol Psychiatry 1998, 39:879-891.

34. Bishop D, Laws G, Adams C, Norbury C: High heritability of speech and language impairments in 6-year-old twins demonstrated using parent and teacher report. Behav Genet 2006, 36:173-184.

35. Whitehouse AJ, Maybery MT, Hart R, Mattes E, Newnham JP, Sloboda DM, Stanley FJ, Hickey M: Fetal androgen exposure and pragmatic language ability of girls in middle childhood: Implications for the extreme malebrain theory of autism. Psychoneuroendocrinology 2010, 35:1259-1264.

36. Williams E, Thomas $\mathrm{K}$, Sidebotham $\mathrm{H}$, Emond A: Prevalence and characteristics of autistic spectrum disorders in the ALSPAC cohort. Dev Med Child Neurol 2008, 50:672-677.

37. Whitehouse AJ, Hickey M, Stanley FJ, Newnham JP, Pennell CE: Brief report: A preliminary study of fetal head circumference growth in autism spectrum disorder. J Autism Dev Disord 2011, 41:122-129.

38. Paternoster L, Zhurov Al, Toma AM, Kemp JP, St Pourcain B, Timpson NJ, McMahon G, McArdle W, Ring SM, Smith GD, Richmond S, Evans DM: Genome-wide association study of three-dimensional facial morphology identifies a variant in PAX3 associated with nasion position. Am J Hum Genet 2012, 90:478-485.

39. Taal HR, St. Pourcain B, Thiering E, Das S, Mook-Kanamori DO, Warrington NM, Kaakinen M, Kreiner-Møller E, Bradfield JP, Freathy RM, Geller F, Guxens M, Cousminer DL, Kerkhof M, Timpson NJ, Ikram MA, Beilin LJ, Bønnelykke K, Buxton JL, Charoen P, Chawes BLK, Eriksson J, Evans DM, Hofman A, Kemp JP, Kim CE, Klopp N, Lahti J, Lye SJ, McMahon G, et al: Common variants at $12 q 15$ and $12 q 24$ are associated with infant head circumference. Nat Genetics 2012, 44:532-538.

40. Price AL, Patterson NJ, Plenge RM, Weinblatt ME, Shadick NA, Reich D Principal components analysis corrects for stratification in genome-wide association studies. Nat Genet 2006, 38:904-909. 
41. Li Y, Willer C, Sanna S, Abecasis G: Genotype imputation. Annu Rev Genomics Hum Genet 2009, 10:387-406.

42. Faraway JJ: Extending the Linear Model with R: Generalized Linear, Mixed Effects and Nonparametric Regression Models. Boca Raton, FL: Chapman \& Hall/CRC; 2006.

43. Devlin B, Roeder K: Genomic control for association studies. Biometrics 1999, 55:997-1004.

44. Willer CJ, Li Y, Abecasis GR: METAL: fast and efficient meta-analysis of genomewide association scans. Bioinformatics 2010, 26:2190-2191.

45. PLINK: Whole genome data analysis toolset. http://pngu.mgh.harvard. edu/ purcell/plink/.

46. Kirkwood BR, Sterne JAC: Essential Medical Statistics, Volume 2nd. Oxford, UK: Blackwell Science; 2003.

47. de Bakker PI, Ferreira MA, Jia X, Neale BM, Raychaudhuri S, Voight BF: Practical aspects of imputation-driven meta-analysis of genome-wide association studies. Hum Mol Genet 2008, 17:R122-R128.

48. Liu JZ, McRae AF, Nyholt DR, Medland SE, Wray NR, Brown KM, AMFS Investigators, Hayward NK, Montgomery GW, Visscher PM, Martin NG, Macgregor S: A versatile gene-based test for genome-wide association studies. Am J Hum Genet 2010, 87:139-145.

49. Yang J, Manolio TA, Pasquale LR, Boerwinkle E, Caporaso N, Cunningham $J M$, de Andrade $M$, Feenstra B, Feingold E, Hayes MG, Hill WG, Landi MT, Alonso A, Lettre G, Lin P, Ling H, Lowe W, Mathias RA, Melbye M, Pugh E, Cornelis MC, Weir BS, Goddard ME, Visscher PM: Genome partitioning of genetic variation for complex traits using common SNPs. Nat Genet 2011, 43:519-525

50. UCSC Genome Bioinformatics. http://genome.ucsc.edu/.

51. RegulomeDB. http://regulome.stanford.edu/.

52. Klei L, Sanders SJ, Murtha MT, Hus V, Lowe JK, Willsey AJ, Moreno-De-Luca D, Yu TW, Fombonne E, Geschwind D, Grice DE, Ledbetter DH, Lord C, Mane SM, Martin CL, Martin DM, Morrow EM, Walsh CA, Melhem NM, Chaste P, Sutcliffe JS, State MW, Cook EH Jr, Roeder K, Devlin B: Common genetic variants, acting additively, are a major source of risk for autism. Mol Autism 2012, 3:9.

53. Plomin R, DeFries JC, Knopik VS, Neiderhiser JM: Behavioral Genetics. 6th edition. New York, NY: Worth Publishers; 2013.

54. Torres AR, Sweeten TL, Cutler A, Bedke BJ, Fillmore M, Stubbs EG, Odell D: The association and linkage of the HLA-A2 class I allele with autism. Hum Immunol 2006, 67:346-351.

55. OMIM - Online Mendelian Inheritance in Man. http://www.omim.org/.

56. Fukushige $S$, Kondo $E, G$, Z, Suzuki H, Horii A: RET finger protein enhances MBD2- and MBD4-dependent transcriptional repression. Biochem Biophys Res Commun 2006, 351:85-92.

57. Cukier HN, Rabionet R, Konidari I, Rayner-Evans MY, Baltos ML, Wright HH, Abramson RK, Martin ER, Cuccaro ML, Pericak-Vance MA, Gilbert JR: Novel variants identified in methyl-CpG-binding domain genes in autistic individuals. Neurogenetics 2010, 11:291-303.

58. Rogers SJ, Hepburn S, Wehner E: Parent reports of sensory symptoms in toddlers with autism and those with other developmental disorders. J Autism Dev Disord 2003, 33:631-642.

59. Wiggins L, Robins D, Bakeman R, Adamson L: Brief report: Sensory abnormalities as distinguishing symptoms of autism spectrum disorders in young children. J Autism Dev Disord 2009, 39:1087-1091.

60. Rosenhall U, Nordin V, Sandström M, Ahlsén G, Gillberg C: Autism and hearing loss. J Autism Dev Disord 1999, 29:349-357.

61. Davies G, Tenesa A, Payton A, Yang J, Harris SE, Liewald D, Ke X, Le Hellard S, Christoforou A, Luciano M, McGhee K, Lopez L, Gow AJ, Corley J, Redmond P, Fox HC, Haggarty P, Whalley LJ, McNeill G, Goddard ME, Espeseth T, Lundervold AJ, Reinvang I, Pickles A, Steen VM, Ollier W, Porteous DJ, Horan M, Starr JM, Pendleton N, et al: Genome-wide association studies establish that human intelligence is highly heritable and polygenic. Mol Psychiatry 2011, 16:996-1005.

62. Robinson EB, Munir K, Munafò MR, Hughes M, McCormick MC, Koenen KC: Stability of autistic traits in the general population: Further evidence for a continuum of impairment. J Am Acad Child Adolesc Psychiatry 2011, 50:376-384.

63. Whitehouse AJ, Hickey M, Ronald A: Are autistic traits in the general population stable across development? PLOS ONE 2011, 6:e23029.

64. Kerin T, Ramanathan A, Rivas K, Grepo N, Coetzee GA, Campbell DB: A noncoding RNA antisense to moesin at 5p14.1 in autism. Sci Trans/ Med 2012, 4:128ra40.
65. Lango Allen H, Estrada K, Lettre G, Berndt SI, Weedon MN, Rivadeneira F, Willer CJ, Jackson AU, Vedantam S, Raychaudhuri S, Ferreira T, Wood AR, Weyant RJ, Segrè AV, Speliotes EK, Wheeler E, Soranzo N, Park J-H, Yang J, Gudbjartsson D, Heard-Costa NL, Randall JC, Qi L, Vernon Smith A, Mägi R, Pastinen T, Liang L, Heid IM, Luan J, Thorleifsson G, et al: Hundreds of variants clustered in genomic loci and biological pathways affect human height. Nature 2010, 467:832-838

66. Benyamin B, St. Pourcain B, Davis OS, Davies G, Hansell NK, Brion MJ, Kirkpatrick RM, Cents RA, Franić S, Miller MB, Haworth CM, Meaburn E, Price TS, Evans DM, Timpson N, Kemp J, Ring S, McArdle W, Medland SE, Yang J, Harris SE, Liewald DC, Scheet P, Xiao X, Hudziak JJ, de Geus EJ, Wellcome Trust Case Control Consortium 2 (WTCCC2), Jaddoe WW, Starr JM, Verhulst FC, Pennell C, et al: Childhood intelligence is heritable, highly polygenic and associated with FNBP1L. Mol Psychiatry 2013. doi: 10.1038/mp.2012.184.

67. Volden J, Phillips L: Measuring pragmatic language in speakers with autism spectrum disorders: Comparing the children's communication checklist-2 and the test of pragmatic language. Am J Speech Lang Pathol 2010, 19:204-212.

doi:10.1186/2040-2392-4-34

Cite this article as: St. Pourcain et al:: Common variation contributes to the genetic architecture of social communication traits. Molecular Autism 2013 4:34.

\section{Submit your next manuscript to BioMed Central and take full advantage of:}

- Convenient online submission

- Thorough peer review

- No space constraints or color figure charges

- Immediate publication on acceptance

- Inclusion in PubMed, CAS, Scopus and Google Scholar

- Research which is freely available for redistribution

Submit your manuscript at www.biomedcentral.com/submit
C) Biomed Central 